\title{
Relation between abnormal patterns of muscle activation and response to common peroneal nerve stimulation in hemiplegia
}

\author{
J H Burridge, D L McLellan
}

\begin{abstract}
Objective-To investigate the relation between response to common peroneal nerve stimulation, timed to the swing phase of walking, and abnormal ankle movement and muscle activation patterns.

Method-Eighteen patients who took part had a drop foot and had had a stroke at least 6 months before the study Twelve age matched normal subjects were also studied. Response to stimulation was measured by changes in the speed and effort of walking when the stimulator was used. Speed was measured over $10 \mathrm{~m}$ and effort by the physiological cost index. Abnormal ankle movement and muscle activation were measured in a rig by ability to follow a tracking signal moving sinusoidally at either 1 or $2 \mathrm{~Hz}$, resistance to passive movement, and EMG activity during both passive and active movements. Indices were derived to define EMG response to passive stretch, coactivation, and ability to activate muscles appropriately during active movement
\end{abstract}

Results-Different mechanisms underlying the drop foot were seen. Results showed that patients who had poor control of ankle movement and spasticity, demonstrated by stretch reflex and coactivation, were more likely to respond well to stimulation. Those with mechanical resistance to passive movement and with normal muscle activation responded less well.

Conclusions-The results support the hypothesis that stimulation of the common peroneal nerve to elicit a contraction of the anterior tibial muscles also inhibits the antagonist calf muscles. The technique used may be useful in directing physiotherapy by indicating the underlying cause of the drop foot.

(F Neurol Neurosurg Psychiatry 2000;69:353-361)

Keywords: functional electrical stimulation; spasticity; hemiplegia

In 1960 Liberson et $a l^{1}$ first used functional electrical stimulation (FES) to improve the gait of hemiplegic patients with a drop foot. Since then FES has aroused enthusiastic but generally sporadic interest in various parts of the world, most consistently Slovenia. ${ }^{2-5}$ Despite the reported benefits it is still not widely used.

Merletti et al $l^{6}$ studied the therapeutic effects of stimulation in a sample of 49 patients, selected from a hemiplegic population of 250 .
The percentage of "excellent" results decreased with time from onset of lesion and severity of spasticity and increased with increasing duration of treatment. Merletti et al concluded that good FES candidates represented about $20 \%$ of the ambulatory hemiparetic population but that careful selection and application was important. No statistical analysis of the results was presented.

A cross over study by Granat et al found an improvement both in gait variables (correction of inversion at the ankle) and in dependency measures (Barthel index) when the common peroneal nerve was stimulated during the swing phase of walking. They concluded that FES is a useful orthotic device for a selected subpopulation of hemiplegic patients. A meta-analysis ${ }^{8}$ identified further evidence to support the use of FES during stroke rehabilitation.

In 1993 a prospective study of a small sample of patients with hemiplegia of vascular origin compared the effects of conventional physiotherapy with physiotherapy that included training with a single channel FES device ${ }^{9}$. The bursts of stimulation were timed, using a foot switch, with the swing phase of the gait cycle. Patients used the stimulator both during physiotherapy and in their everyday life. The group of patients were monitored while using the stimulator during a period of 3 months after which significant improvement in speed and physiological cost of walking (measured by physiological cost index) were shown when the stimulator was used. However, closer examination of these results showed that, although 11 patients experienced more than $10 \%$ improvement in walking variables two had minimal benefit and three none at all. Failure of one third of apparently suitable patients to benefit could perhaps explain why FES has not become more widely used. It also raises the question as to whether closer analysis of the pattern of muscular activation in patients with foot drop due to upper motor neuron lesions could improve the selection of suitable patients and thus reduce the failure rate.

Patients with hemiplegia as a result of an upper motor neuron lesion are often assumed to have all the characteristic features of spasticitybrisk tendon reflexes including clonus and an increased tonic stretch reflex. Other components of the upper motor neuron syndrome, such as weakness of voluntary muscular activation, inappropriate activation, or poor voluntary control are often if not invariably present. A third set of potential factors relate to the mechanical changes in the muscle associated 
with immobility and increased tone-notably, increased stiffness during stretch and contracture of muscle fibres and increased stiffness of soft tissues around the joints.

One of the difficulties in evaluating the clinical relevance of spasticity is that although active voluntary movements (such as walking) are the target of clinical assessment and treatment, spasticity is usually measured by reference to the excitability or strength of passive movements applied to the affected muscles, such measurements being made while the patient lies in a relaxed position. Detailed studies of the relation between the activation of lengthening muscles under these two conditions have shown that there is considerable variability from one patient to another even when conventional classic testing has suggested that the patients are similar. Neural activation of the lengthening muscle during passive movement (the stretch response) may be greater than, the same as, or less than its activation during active voluntary movement (inappropriate coactivation) when it causes what Knutsson and Martensson have termed "spastic restraint". ${ }^{10}$ Moreover, antispasticity medication may suppress passive stretch responses without affecting restraint during voluntary movement ${ }^{11}$ and may indeed enhance agonist activation rather than reduce spastic restraint in certain people. For this reason it is important to demonstrate the degree of inappropriate coactivation that causes spastic restraint during voluntary movements and not to assume that what is detected during passive examination will necessarily reflect what is happening during functional movements. ${ }^{12}$

In the study reported here, muscle activation of the anterior tibial and calf muscles during both passive and active movement, control of voluntary movement, and resistance to passive movement were all measured and related to clinical response to FES.

\section{Method}

SUBJECT SELECTION

Patients with residual hemiplegia after stroke (more than 6 months after onset) who had been referred for treatment with FES were invited to take part. As in the prospective study ${ }^{9}$ patients were selected who were unable to achieve heel strike when walking, were able to walk $10 \mathrm{~m}$, and had no serious medical conditions likely to influence response to treatment. Twelve age matched normal subjects were also studied. Informed consent was obtained from all subjects and ethics committee approval had been granted.

FUNCTIONAL ELECTRICAL STIMULATION

Ankle dorsiflexion was produced during the swing phase of walking, through stimulation of the common peroneal nerve. One electrode, usually the cathode, was positioned on the skin, close to the nerve as it passes around the head of the fibula. The second electrode, usually the anode, was positioned either on the motor point of the tibialis anterior, slightly lateral to this, or occasionally in the popliteal fossa. Electrode positions were adjusted so that ankle movement and heel strike were, by observation, as close to "normal" as possible. Stimulation was initiated and terminated to synchronise with the swing phase of walking using a force sensitive resistor switch, positioned either under the heel or the first metatarsal head of the foot on the hemiplegic side. Adjustment of the rate of rise of the amplitude of the stimulation pulses allowed active dorsiflexion to occur when toe lift was needed. The common peroneal stimulator used in the study was the

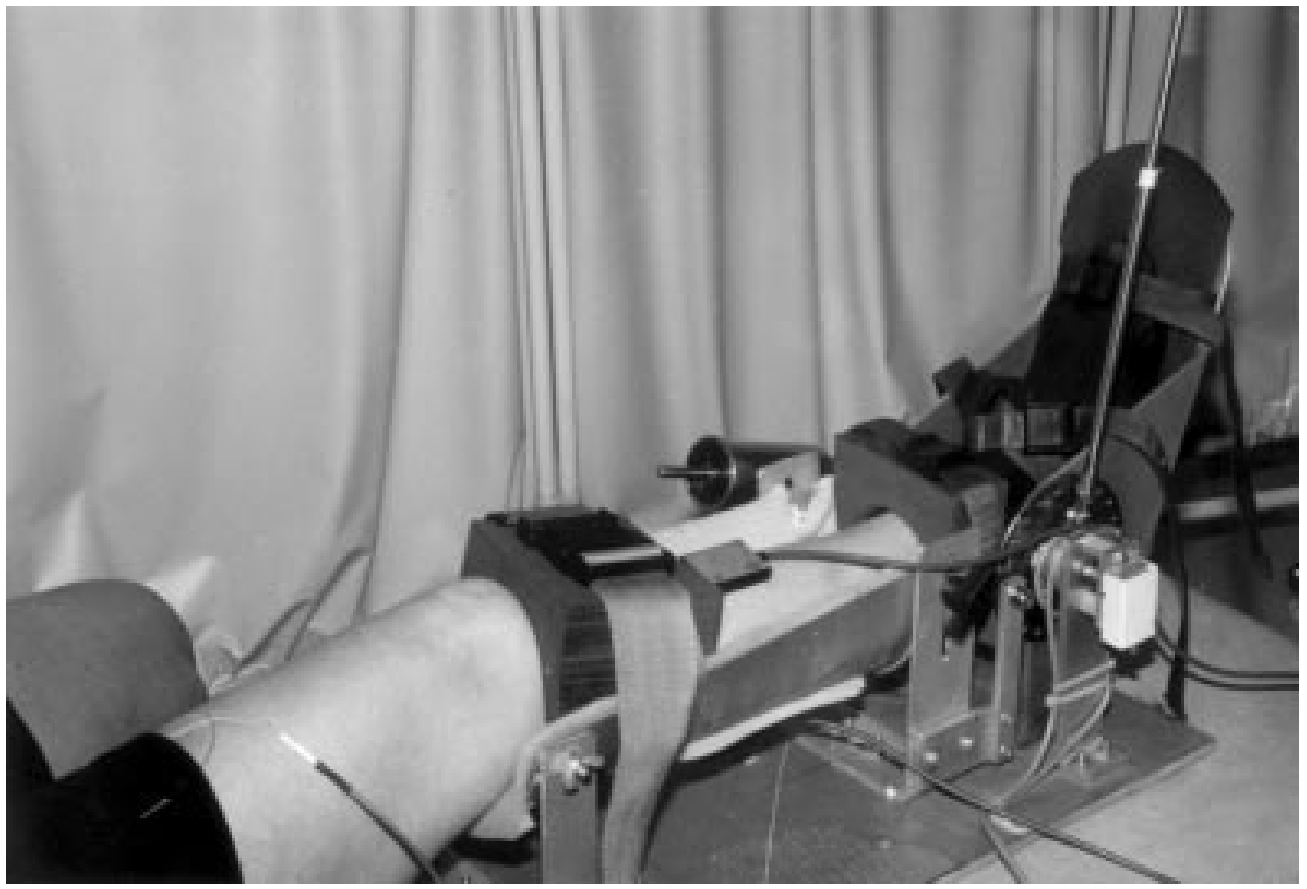

Figure 1 Photograph of the ankle rig showing the position of the leg, electrodes, potentiometer, strain gauge, counterbalance, and lever. 
ODFS II. This delivers biphasic asymmetric and charge balanced pulses at $300 \mu$ s with an output of $0-85 \mathrm{~mA}$ into a $1 \mathrm{k} \Omega$ load at a frequency of $40 \mathrm{~Hz}$. PALS 3.6 or $5.0 \mathrm{~cm}$ electrodes were used.

\section{MEASUREMENT OF MUSCLE ACTIVATION} PATTERNS

A rig was built to monitor the angle and resistance to passive movement of the ankle joint, while allowing simultaneous recording of electrical signals from the muscles acting across the joint (fig 1). The rig supported the lower leg and foot, and was hinged so that the ankle could be moved in the sagittal plane either passively (by the operator moving the lever attached to the fulcrum) or actively, by the subject.

A computer generated elliptical tracking signal, oscillating in simple harmonic motion at a frequency of 1 or $2 \mathrm{~Hz}$, with an amplitude of 30 degrees, was displayed on a computer screen. Ankle angle, measured by a potentiometer, was also displayed on the screen in the form of a cross. When the movement of the ankle matched the oscillation of the tracking signal the cross appeared within the ellipse. To reinforce accurate tracking the cross changed colour, green when it was within the ellipse, amber when partially within it, and red when completely outside it. A strain gauge on the lever measured the torque $(\mathrm{Nm})$ exerted when the ankle was moved passively. The voltage from this and the goniometer were recorded digitally on videotape through a Softel datalog recorder. Torque and angle were calibrated with voltage from the strain gauge and potentiometer and with Softel units.

EMG RECORDING AND PROCESSING

Location of electrodes: The electrode over the anterior tibial muscle was located $1.5 \mathrm{~cm}$ from the anterior border of the tibia at the junction between the upper and middle third of a line between the tibial tubercle and the intermalleolar line. The electrode over the calf muscles was located $1.5 \mathrm{~cm}$ lateral to a line between the tendoachilles and the knee crease at the junction between the upper and middle thirds. In this position signals arising from the lower part of the lateral head of the gastrocnemius and from soleus would have been detected.

Surface EMG signals were recorded using Medelec contact electrodes, with a common mode rejection at $50 \mathrm{~Hz}$ of approximately 100 dB. Frequencies between 10 and $1000 \mathrm{~Hz}$ were received and amplified $\times 15$. The signal was transmitted to the main amplifier at low impedance. In the main amplifier the calf channel was further amplified $\times 470$ and the tibialis anterior channel $\times 47$. The signal was rectified then integrated by a low pass filter, using a $2.2 \mu \mathrm{f}$ capacitor (time constant 13.8 seconds). Both the raw and integrated EMG signals were digitised, recorded on videotape using the Softel system, and saved for reference together with the torque, goniometer, and tracking signal data. There were therefore seven channels recording in real time on videotape at a sample rate of 500/s.
ADJUSTING THE RIG FOR INDIVIDUAL SUBJECTS Tests were performed through an arc of 30 degrees with all subjects, but the starting position was adjusted so that it corresponded with the middle of each subject's active range. This was defined as the mean of three measurements taken at the start of the first baseline test. The weight of the footplate was counterbalanced by a lead weight on an arm attached to the side of the rig. The position of the weight was adjusted for each subject to correct for variations in mass and length of the foot.

PASSIVE AND ACTIVE TASKS

During the passive test the ankle was moved at $2 \mathrm{~Hz}$ and the assessor ensured that the tracking signal followed precisely the ellipse displayed on the screen. Subjects provided neither voluntary assistance nor resistance. The active test was performed at 1 and $2 \mathrm{~Hz}$. Subjects were asked to follow the tracking signal as accurately as possible. Sufficient practice time was allowed for them to feel that they had mastered the task as well as they were able.

\section{RETRIEVING DATA}

Video recordings of the tests were replayed and samples were selected for analysis. The accuracy of tracking varied during the active test; therefore, the samples used for analysis were taken from each subject's best performance, defined as the best match between target trace and tracking signals. In the passive test, the earliest accurate samples were used because in certain subjects responses showed some habituation after many repetitions. Three samples, each lasting 5 seconds, were retrieved from each test and analysed. The degree of interrater and intrarater error was tested and found to be reliable.

ANALYSIS OF THE ANKLE RIG DATA AND

CALCULATION OF THE INDICES

Samples of data, retrieved from the video recordings, were analysed in Microsoft Excel using a macro written in visual basic.

\section{DERIVATION OF THE INDICES USING THE EMG}

SIGNALS

Problems are encountered when comparing EMG amplitudes in different subjects and to a lesser extent in the same subject on different occasions. Electrode contact, thickness and condition of the skin, depth of subcutaneous fat, and proximity of the electrode to the muscle motor point all influence the amplitude of the signal. Using the ratio of EMG activity during two defined periods under controlled conditions overcomes these problems, showing greater consistency than the absolute EMG signals in repeated measurements. ${ }^{11}{ }^{12}$ Ratios to quantify muscle activity during defined phases of movement were therefore derived and are described below. Although raw and root mean squared (RMS) EMG signals were recorded and checked visually for the presence of artefacts, only the RMS envelope was used in the analysis. For each RMS sample analysed the minimum value was determined and used 


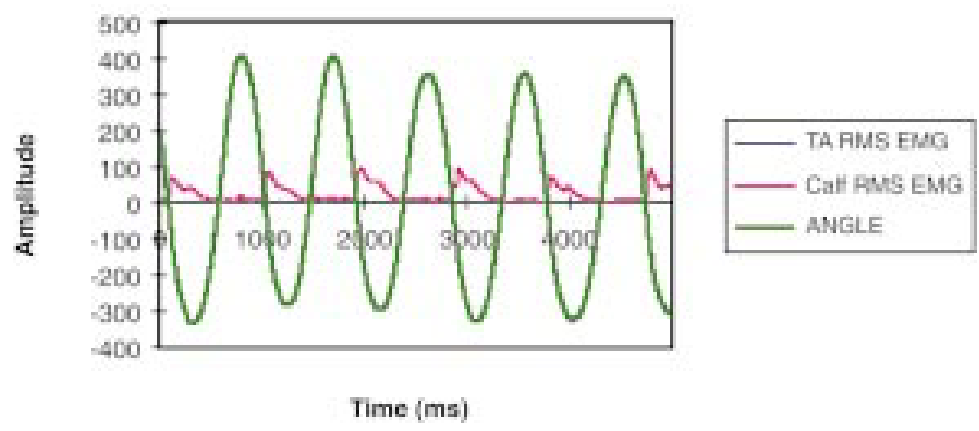

Figure 2 Calculation of the calf muscles' stretch index (SI). RMS calf EMG was summed from peak plantarflexion (positive peak) to peak dorsiflexion (negative peak)-stretch phase, and from peak dorsiflexion (negative peak) to peak plantarflexion (positive peak)-shortening phase. The same method was used to calculate the TA stretch index. In this example of a spastic patient a stretch index is seen in the calf muscles but EMG activity in the tibialis anterior did not reach the threshold for recording. In the normal subject the index is roughly 1 because in the relaxed state there is little or no muscular response. Where there is a reflex response to stretch it is $>1$. Calf SI was 2.34, anterior tibial stretch index $=0.96$. (100 softel units $=1.4 \mathrm{mV}$. Calf EMG is amplified $\times 7050$, anterior tibial $E M G \times 705)$.

as the zero reference. The following values and indices were derived to describe the subject's performance in the passive and active tasks.

PASSIVE TASK

Peak torque was recorded when the ankle was dorsiflexed (TQ). The stretch index was derived (fig 2) to quantify the reflex muscle activity in the calf muscles, experienced by some hemiplegic patients, during passive stretch (SI) at $2 \mathrm{~Hz}$ through 30 degrees, as described above.

Stretch index $(\mathrm{SI})=$

Sum of calf muscle EMG activity in the stretch phase Sum of calf muscle EMG activity in the shortening phase

ACTIVE TASK

Voluntary tracking (VT)

Accuracy in following the tracking signal (VT) was defined as the ratio between the peak excursion of the tracking and ankle angle signals (fig 3 ).

Calf modulation index (CMI)

A normal subject who has no neurological impairment demonstrates reciprocal activation of the calf and anterior tibial muscles when following the sinusoidal trace, so that, for example when the ankle moves from dorsiflexion to plantarflexion the calf muscles are active and the

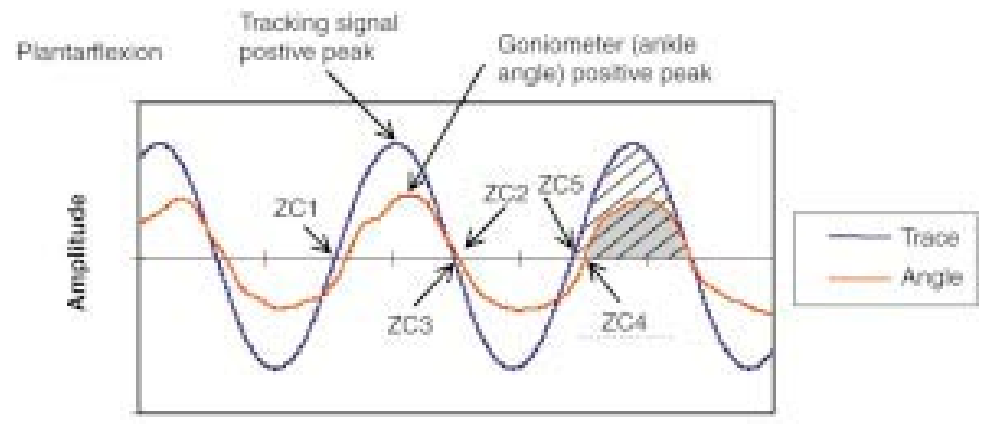

Dersitienon

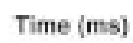

Figure 3 Trace and goniometer signals, the zero crossing points, and peaks from which the tracking index was derived. tibialis anterior is silent. During movement from plantarflexion to dorsiflexion the calf muscles are silent whereas the tibialis anterior is active. In normal subjects, although most activity of the tibialis anterior was confined to the dorsiflexion phase, activation of the calf muscles was sometimes seen after peak plantarflexion - at the beginning of dorsiflexion. To allow for this the CMI was defined as the ratio:

Sum of calf EMG activity from midposition to peak dorsiflexion Sum of calf EMG activity from midposition to peak plantarflexion

(figs 4,5 ).

Tibialis anterior modulation index (TAMI)

The ratio TAMI =

Sum of tibialis anterior EMG activity from peak dorsiflexion to peak plantarflexion

Sum of tibialis anterior EMG activity from peak plantarflexion to peak dorsiflexion

was used to identify pattern of activity in the anterior tibial muscles during active ankle movement. The charts of active ankle movement and EMG activity showed no subjects, either normal or hemiplegic, with more than minimal coactivation between the anterior tibial and calf muscles during plantarflexion. A high TAMI was therefore considered to represent poor activation of the anterior tibial muscles during dorsiflexion.

DESIGN OF THE STUDY

An $A B$ case study design was used. After recruitment, subjects were assessed three times over a period of between 3 and 4 weeks (period A). Baseline values were therefore the mean of three prestimulation tests. A single channel FES device was then set up and adjusted. Subjects were then reassessed after 1 and 3 months of continuous use of the stimulator (period B).

OUTCOME MEASURES

The response to stimulation was measured by changes in walking speed and physiological cost index (PCI), a validated measure of the effort of walking defined by $\mathrm{Nene}^{13}$ as:

Heart rate after walking-resting heart rate (beats/min) Walking speed $(\mathrm{m} / \mathrm{min})$

Measurements were taken both with and without stimulation. Subjects were tested over a $10 \mathrm{~m}$ walk. Two metres were allowed at either end of the walkway for acceleration and deceleration. The average of three consecutive measurements was recorded, the first two measurements were made without stimulation followed by three with and ending with one without. Measurements were taken at each baseline assessment, when the stimulator was first set up, and after 1 and 3 months of continued use.

STATISTICAL ANALYSIS

Data were not normally distributed and therefore non-parametric tests were used to analyse continuous variables. Median as well as mean values have been quoted. Relations between normal subjects and hemiplegic patients and between variables within these groups were 


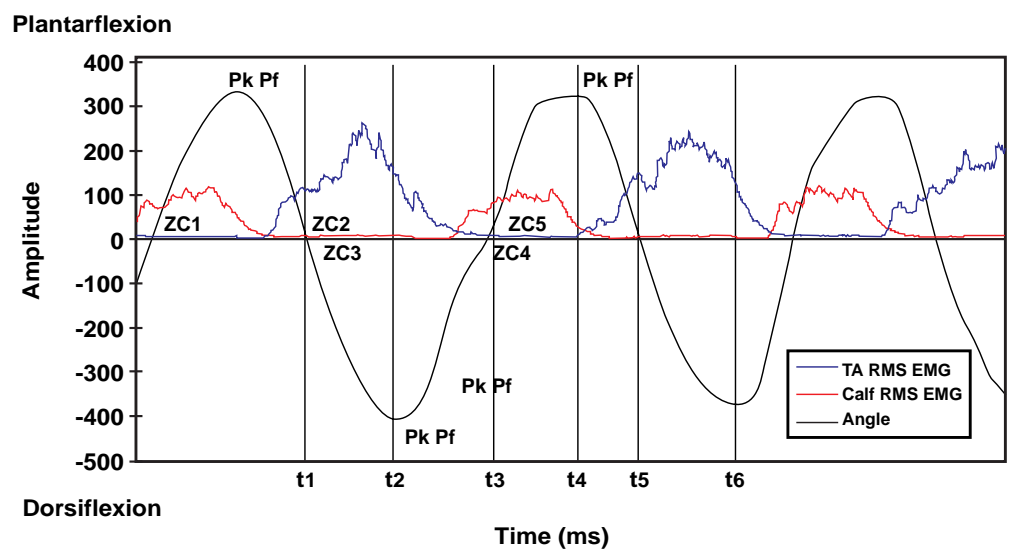

Figure 4 Method for deriving the calf modulation index. Calf RMS EMG was integrated from ZC2 (zero crossing 2) to peak dorsiflexion (negative peak) $\left(t_{1}-t_{2}\right)$ - relaxation phase - and from $Z C 4$ to peak plantarflexion (positive peak) $\left(t_{3}-t_{4}\right)$-active phase. TA EMG was integrated from $t_{3}-t_{4}$ (relaxation phase) and $t_{5}-t_{6}$ (active phase). A normal pattern of reciprocal activation is seen in this example (100 softel units $=1.4 \mathrm{mv}$. Calf EMG is amplified $\times 7050$, anterior tibial $E M G \times 705)$.

\section{Plantarflexion}

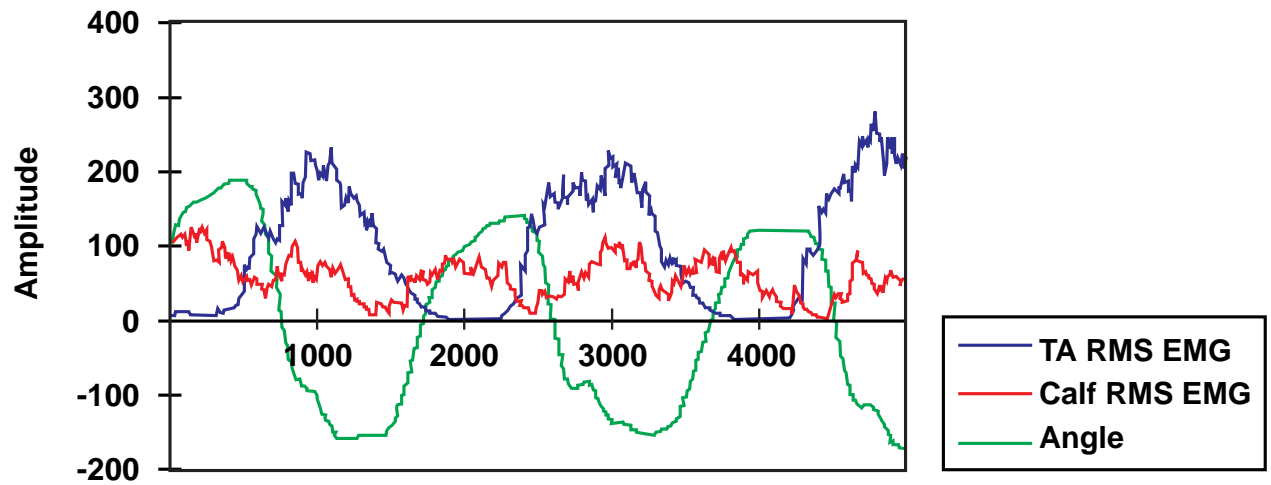

\section{Dorsiflexion}

Time (ms)

Figure 5 Example of coactivation of the calf with the anterior tibial muscles during dorsiflexion, but normal reciprocal inhibition of the anterior tibial muscles during plantarflexion (100 softel units $=1.4 \mathrm{mV}$. Calf EMG is amplified $\times 7050$, anterior tibial $E M G \times 705)$.

measured using Spearman's rank correlation coefficient. Data from the normal sample were compared with data from the hemiplegic sample using the Mann-Whitney $U$ test and data from within each sample, using the Wilcoxon signed ranks test. Where appropriate $95 \%$ confidence intervals have been quoted.

Hemiplegic patients were grouped into three categories on the basis of their muscle activation patterns. Increasing abnormality was represented by an increase in SI, TQ CMI, and TAMI and a decrease in VT. Where the index fell within the last quartile the abnormality was classed as severe. More specific classification was considered inappropriate because of the small sample size and lack of previous experimental evidence.

\section{Results}

SUBJECTS

Eighteen hemiplegic patients were recruited and data were also collected from 12 age matched normal subjects (table 1). All the hemiplegic patients who were recruited completed the baseline and 3 month assessment period.
RESULTS OF ANKLE RIG TESTS

Analysis of the data from all the subjects indicated that the cause of drop foot was not necessarily an inability to activate the ankle dorsiflexors during walking. Different types of abnormal muscle activation were identified in different people, but people were not seen to change from one type to another.

RELATION BETWEEN INDICES AND RAW DATA

Throughout the data collection period the indices that were derived from the raw data were compared with the video data and graphs of the raw data, to ensure that the indices represented a true reflection of the observed movement and pattern of muscle activity. Data collected from the normal subjects were used to define the normal range for each of the indices.

COMPARISON OF HEMIPLEGIC AND NORMAL DATA Values measured and indices derived identified significant differences between hemiplegic patients and normal subjects in all baseline performance measures except the TAMI measured at $1 \mathrm{~Hz}$. The differences between the 
Table 1 Demographic data for the hemiplegic and normal subjects

\begin{tabular}{|c|c|c|c|c|c|}
\hline & Mean age at start of trial & $\operatorname{Sex}$ & Cause of stroke & $\begin{array}{l}\text { Side of } \\
\text { hemiplegia }\end{array}$ & $\begin{array}{l}\text { Mean time from } \\
\text { stroke }\end{array}$ \\
\hline Patients & 60 y 10 months & $\begin{array}{l}11 \text { Male } \\
7 \text { Female }\end{array}$ & $\begin{array}{l}10 \text { Infarction } \\
3 \text { Haemorrhage } \\
4 \text { Unknown }{ }^{\star} \\
1 \text { Multiple emboli }\end{array}$ & $\begin{array}{l}\text { 10 Right } \\
8 \text { Left }\end{array}$ & 2 y 11 months \\
\hline Age range & 47 y 5 months -76 y 2 months & & & & 3 y 2 months \\
\hline Normal subjects & 55 y 6 months & $\begin{array}{l}5 \text { Male } \\
7 \text { Female }\end{array}$ & & & \\
\hline Age range & 30 y 6 months -73 y 8 months & & & & \\
\hline
\end{tabular}

^In these cases there was no CT. Haemorrhage was not distinguished from infarct.

two groups are shown in table 2 , and illustrated in the charts shown in fig 6 .

CRITERIA USED TO SORT SUBJECTS INTO

PATHOLOGICAL CATEGORIES

For each baseline performance measure, subjects' responses were defined as either normal or pathological. Five categories were identified (table 3).

- Failure to follow the tracking signal (measured by the ratio between the tracking signal and goniometer at peak dorsiflexion during active movement at $1 \mathrm{~Hz}$ ) (VT)

- Excessive coactivation of the calf and anterior tibial muscles during active dorsiflexion (identified in the active test at $1 \mathrm{~Hz}$ by the calf modulation index) (fig 6).

- Failure to modulate the activity of the anterior tibial muscles appropriately (identified in the active test at $1 \mathrm{~Hz}$ by the tibialis anterior modulation index) (TAMI)

- Excessive resistance to passive movement (measured as the peak torque when the ankle was moved passively into dorsiflexion at $2 \mathrm{~Hz}$ ) (TQ)

- Excessive stretch response (measured by the stretch index during passive movement at 2 $\mathrm{Hz}$ ) (SI) (fig 4).

CLASSIFICATION OF SUBJECTS BY ABNORMAL PATTERNS OF MUSCLE ACTIVITY

Abnormal types of muscle activity that may have affected the patients' ability to dorsiflex the ankle when walking or to respond to stimulation were identified. Some patients had one whereas others had multiple abnormalities. It was not possible to identify a predominant abnormality in every case.

Table 2 Median, mean, and SD of variables and indices measured with normal subjects and hemiplegic patients.

\begin{tabular}{|c|c|c|c|c|c|c|c|c|}
\hline & \multicolumn{2}{|l|}{$V T$} & \multicolumn{2}{|l|}{$C M I$} & \multicolumn{2}{|l|}{ TAMI } & \multirow[b]{2}{*}{$T Q$} & \multirow[b]{2}{*}{$S I$} \\
\hline & $(1 \mathrm{~Hz})$ & $(2 \mathrm{~Hz})$ & $(1 \mathrm{~Hz})$ & $(2 \mathrm{~Hz})$ & $(1 \mathrm{~Hz})$ & $(2 \mathrm{~Hz})$ & & \\
\hline \multicolumn{9}{|c|}{ Normal subjects: } \\
\hline Median & 1.03 & 0.93 & 0.10 & 0.07 & 0.31 & 0.21 & 2.75 & 0.96 \\
\hline Mean & 1.10 & 0.90 & 0.10 & 0.08 & 0.30 & 0.21 & 2.77 & 0.96 \\
\hline SD & 0.21 & 0.17 & 0.06 & 0.03 & 0.09 & 0.11 & 0.59 & 0.15 \\
\hline $\mathrm{n}$ & 12 & 12 & 12 & 12 & 12 & 12 & 10 & 10 \\
\hline \multicolumn{9}{|c|}{ Hemiplegic patients: } \\
\hline Median & 0.52 & 0.41 & 0.56 & 0.69 & 0.32 & 0.39 & 10.93 & 1.44 \\
\hline Mean & 0.45 & 0.38 & 0.92 & 0.80 & 0.37 & 0.51 & 12.52 & 1.60 \\
\hline SD & 0.23 & 0.21 & 0.90 & 0.50 & 0.25 & 0.62 & 6.43 & 0.63 \\
\hline $\mathrm{n}$ & 18 & 18 & 18 & 18 & 18 & 18 & 18 & 18 \\
\hline $\begin{array}{l}\text { Mann-Wh } \\
\text { value }\end{array}$ & $<0.001$ & $<0.001$ & $<0.001$ & $<0.001$ & 0.574 & 0.023 & $<0.001$ & 0.002 \\
\hline
\end{tabular}

$\mathrm{VT}=$ voluntary tracking (accuracy in following tracking the target signal); CMI=calf modulation index (coactivation during active movement) ; TAMI=tibialis anterior modulation index (the ability to activate the anterior tibial muscles during ankle dorsiflexion); $\mathrm{TQ}=$ torque (peak resistance measured during passive ankle dorsiflexion); $\mathrm{SI}=$ stretch index (response of the calf muscles to passive stretching). Significant values are shown in bold.
This method of categorisation allowed the following inferences to be drawn:

All subjects who were able to follow the tracking signal were also able to activate their anterior tibial muscles appropriately, but not vice versa. Other factors such as coactivation and mechanical resistance to movement may therefore influence the subjects' ability to follow the tracking signal.

Inability to activate the anterior tibial muscles appropriately (nine patients) was in each case associated with other abnormalities such as inability to follow the tracking signal or coactivation; as illustrated in table 4 .

Ten patients demonstrated either an excessive stretch response or a mechanical resistance to movement, and three had both. In any of the patients where resistance to passive movement was identified it may have contributed to the dropped foot, but for patients 14 and 15, for whom this was the only abnormality detected to explain their inability to follow the tracking signal, it may have been the predominant factor.

ADDITIONAL FINDINGS

Fifteen of the 18 patients studied had an abnormal pattern of muscle activation that may have accounted for their drop foot. Patients 1 and 8 seemed to have no abnormality and, although patient 5 was not able to follow the tracking signal, no explanation for this was identified by the ankle rig test.

Eight subjects had a normal response to passive movement,

DISTINGUISHING COACTIVATION FROM STRETCH REFLEX

In some patients both coactivation and a stretch reflex in the calf muscles were seen. Two patients in whom coactivation was present at $1 \mathrm{~Hz}$ and a passive stretch response at $2 \mathrm{~Hz}$ were tested passively at $1 \mathrm{~Hz}$. In both cases no passive stretch reflex was found at $1 \mathrm{~Hz}$. It is not possible to say whether the coactivation was due to a stretch response the gain of which had been increased in the process of voluntary activation or whether it represented central coactivation. Isometric testing would be needed to resolve this question. ${ }^{14}$

CROSS TALK

A further possibility was that the observed coactivation was an artefact due to cross talk between the two sets of surface electrodes. One patient in whom coactivation was seen with surface electrodes was also studied using 

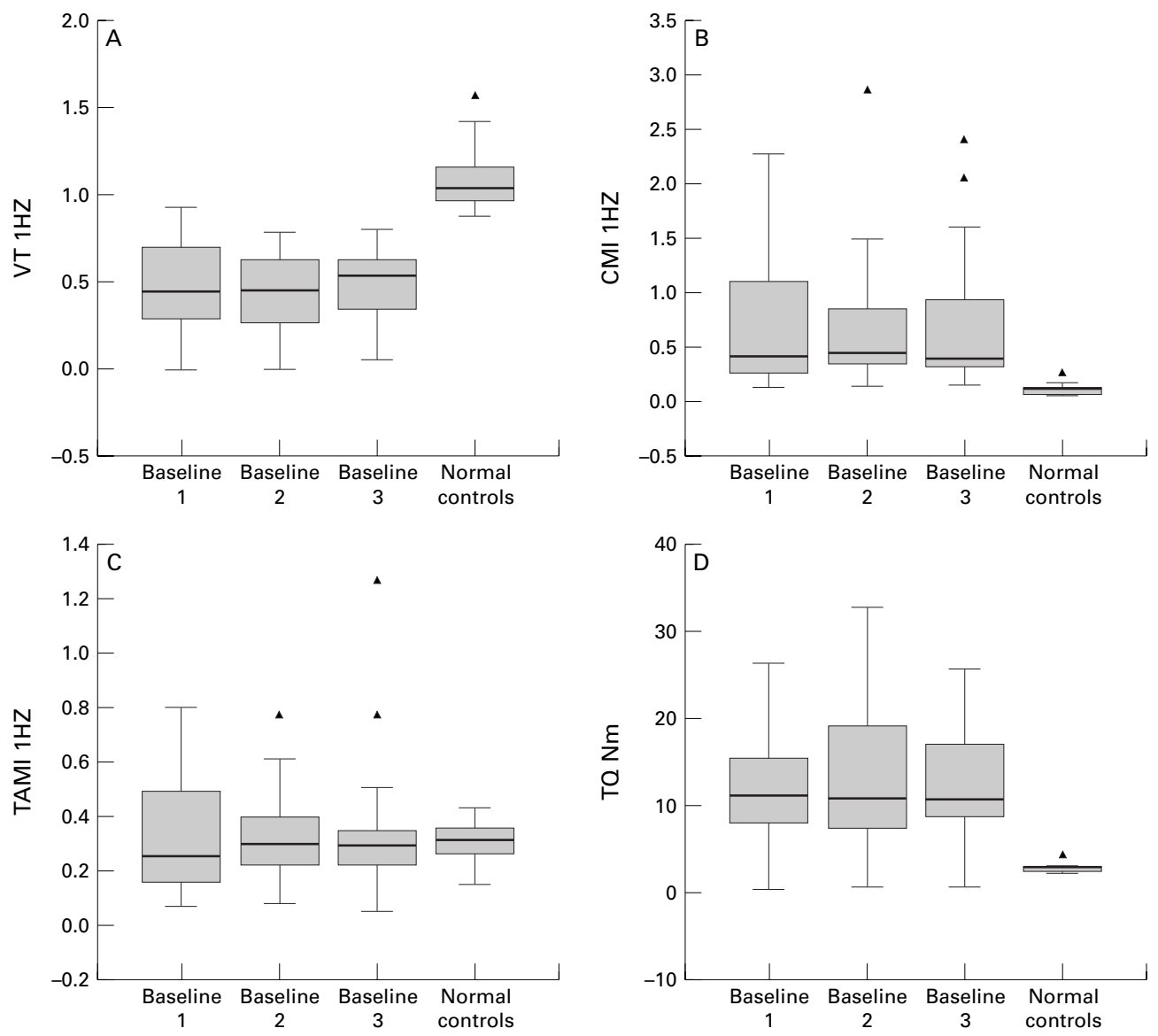

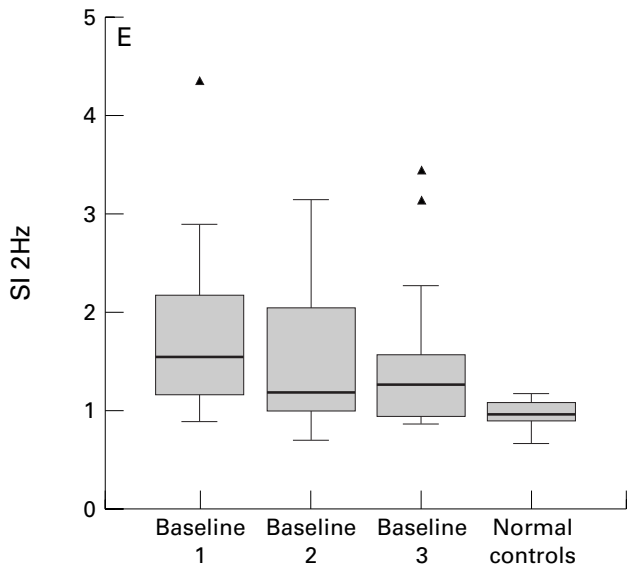

intramuscular needle electrodes placed in the tibialis anterior and soleus muscles. The presence of calf muscle EMG during active dorsiflexion at both $1 \mathrm{~Hz}$ and 2 was confirmed.

CORRELATION BETWEEN BASELINE VARIABLES Statistical analysis, using ranked correlation coefficients, suggested that certain baseline performance measures were covariables (table $4)$. These are summarised as follows:

TAMI and VT

This suggests that the ability to activate the anterior tibial muscles appropriately was an important factor in control of voluntary movement independent of other abnormalities.
Figure 6 Median and range of values for each of the indices, measured at each baseline assessment and in the normal subjects. (A) Voluntary tracking at $1 \mathrm{~Hz},(B)$ Calf index at $1 \mathrm{~Hz}$, (D) Torque (passive test) at $2 \mathrm{~Hz},(E)$ Stretch index (passive test) at $2 \mathrm{~Hz}$.

\section{$T Q$ and $V T$}

This suggests that ankle stiffness hindered control of voluntary movement.

Baseline physiological cost index and VT coactivation, and TAMI

This suggests that impaired voluntary performance and inappropriate calf activity during movement increases the physiological cost of walking.

\section{RESULTS OF OUTCOME MEASURES}

Changes in walking speed and physiological cost index

A statistically significant increase in walking speed and reduction in physiological cost index was measured when the stimulator had been used for 3 months. Patients experienced what 
Table 3 Summary of the categorisation of each subject. For each performance measure a value greater than the median indicated an abnormality and is shown as *. Values in the last quartile (severe abnormality) are shown as **

\begin{tabular}{|c|c|c|c|c|c|}
\hline Subject & $V T$ & $C M I$ & $T A M I$ & $T Q$ & $S I$ \\
\hline 1 & - & - & - & - & - \\
\hline 2 & $\star$ & $\star \star$ & $\star \star$ & - & - \\
\hline 3 & $\star$ & $\star \star$ & - & - & $\star$ \\
\hline 4 & - & $\star$ & - & - & - \\
\hline 5 & $\star \star$ & - & - & - & - \\
\hline 6 & $\star$ & $\star$ & $\star$ & - & - \\
\hline 7 & - & - & - & - & $\star \star$ \\
\hline 8 & - & - & - & - & - \\
\hline 9 & $\star$ & - & - & - & $\star \star$ \\
\hline 10 & $\star \star$ & - & $\star \star$ & $\star \star$ & $\star$ \\
\hline 11 & $\star \star$ & - & $\star \star$ & $\star \star$ & - \\
\hline 12 & - & $\star \star$ & - & $\star \star$ & - \\
\hline 13 & $\star$ & $\star \star$ & $\star \star$ & $\star$ & $\star \star$ \\
\hline 14 & $\star$ & $\star(2 \mathrm{~Hz})$ & - & $\star \star$ & - \\
\hline 15 & $\star$ & - & - & $\star$ & - \\
\hline 16 & $\star$ & $\star$ & * & - & - \\
\hline 17 & $\star \star$ & $\star$ & * & $\star$ & $\star \star$ \\
\hline 18 & $\star$ & $\star$ & $\star$ & - & - \\
\hline
\end{tabular}

might be described as an orthotic effect, measured by improvement in these variables only when the stimulator was used at the fifth, 3 month assessment, and a therapeutic effect, evidenced by improvement even when not using the stimulator-after 3 months of using it. Median and percentage changes and results of the statistical tests are shown in table 5 .

Relation between baseline performance measures and response to stimulation

Statistical tests (table 4) indicated that the following baseline performance measures were associated with an increase in walking speed with stimulation and could thus be classed as a predictor of variables:

- An inability actively to dorsiflex the ankle

- Calf coactivation at $1 \mathrm{~Hz}$
- Excessive stretch response (SI)

- A low baseline walking speed concurrently with a high baseline physiological cost index

Calf coactivation predicted an increased physiological cost of walking (physiological cost index).

\section{SUMMARY OF RESULTS}

(1) For the group as a whole treatment with FES was associated with an increase in walking speed and a decrease in physiological cost of walking.

(2) The greatest increase in speed of walking was seen in people who had difficulty following a tracking signal and in whom inappropriate coactivation occurred during this task.

(3) Not all those who had a marked resistance to passive movement benefited from FES. Those who did not benefit tended to be those who had mechanical stiffness rather than spasticity (increased stretch reflex and/or coactivation during voluntary dorsiflexion).

(4) The mechanisms underlying foot drop were varied. Foot drop in poststroke hemiplegia could in different patients be associated with one or more of the following:

- Coactivation of calf and anterior tibial muscles during active dorsiflexion

- Selective activation in the anterior tibial muscles

- Failure to modulate actively the anterior tibial and/or calf muscles

- A normal response to passive dorsiflexion of the ankle.

(5) There is a trend for those with an excessive stretch response on EMG to have a high passive torque but either can occur

Table 4 Matrix of ranked correlation coefficients

\begin{tabular}{|c|c|c|c|c|c|c|c|c|c|}
\hline & $\begin{array}{l}V T \\
(1 \mathrm{~Hz})\end{array}$ & $\begin{array}{l}C M I \\
(1 \mathrm{~Hz})\end{array}$ & $\begin{array}{l}\text { TAMI } \\
(1 \mathrm{~Hz})\end{array}$ & Torque & SI & BSpeed & $B P C I$ & $\begin{array}{l}\text { Speed } \\
\text { S5-NS5 }\end{array}$ & $\begin{array}{l}P C I \\
\text { S5-NS5 }\end{array}$ \\
\hline VT $(1 \mathrm{~Hz})$ & & 0.420 & 0.668 & 0.523 & 0.271 & 0.404 & 0.690 & -0.721 & -0.065 \\
\hline CMI $(1 \mathrm{~Hz})$ & & & 0.486 & 0.368 & 0.358 & 0.152 & 0.519 & -0.658 & -0.459 \\
\hline TAMI (1Hz) & & & & 0.430 & 0.197 & 0.057 & 0.381 & -0.377 & -0.218 \\
\hline TQ & & & & & 0.381 & 0.340 & 0.302 & -0.327 & -0.253 \\
\hline SI & & & & & & -0.125 & 0.044 & -0.459 & -0.123 \\
\hline BSpeed & & & & & & & 0.649 & -0.488 & 0.100 \\
\hline BPCI & & & & & & & & -0.760 & -0.255 \\
\hline S5-NS5 & & & & & & & & & 0.105 \\
\hline S5-NS5 & & & & & & & & & \\
\hline
\end{tabular}

Correlation coefficients that were significant at the 0.05 level are shown in bold script. The table illustrates where there was a correlation between baseline variables and between baseline variables and response to stimulation. PBI=physiological cost index; $\mathrm{S}=$ measurements made with stimulation; $\mathrm{NS}=$ measurements made without stimulation; $\mathrm{B}=$ average baseline measurement; $5=5$ th assessment (after the stimulator had been used for 3 months)

Table 5 Walking speed and PCI measurements at baseline and assessment 5 (3 months).

\begin{tabular}{|c|c|c|c|c|c|c|}
\hline & \multicolumn{3}{|l|}{$P C I$} & \multicolumn{3}{|l|}{ SPEED } \\
\hline & Baseline (NS) & Assess 5 (NS) & Baseline (NS) & Baseline (NS) & Assess 5 (NS) & Baseline (NS) \\
\hline \multicolumn{7}{|l|}{ Measurements at: } \\
\hline Median & 0.68 & 0.55 & 0.68 & 0.57 & 0.67 & 0.57 \\
\hline Mean & 0.88 & 0.64 & 0.88 & 0.63 & 0.74 & 0.63 \\
\hline SD & 0.81 & 0.39 & 0.81 & 0.27 & 0.32 & 0.27 \\
\hline Compared with: & $\begin{array}{l}\text { Assess } 5 \text { (NS) } \\
\text { (therapeutic effect) }\end{array}$ & $\begin{array}{l}\text { Assess } 5 \text { (S) } \\
\text { (orthotic effect) }\end{array}$ & $\begin{array}{l}\text { Assess } 5(S) \\
\text { (combined effect) }\end{array}$ & $\begin{array}{l}\text { Assess } 5 \text { (NS) } \\
\text { (therapeutic effect) }\end{array}$ & $\begin{array}{l}\text { Assess } 5 \text { (S) } \\
\text { (orthotic effect) }\end{array}$ & $\begin{array}{l}\text { Assess } 5(S) \\
\text { (combined effect) }\end{array}$ \\
\hline Median & 0.55 & 0.50 & 0.50 & 0.67 & 0.71 & 0.71 \\
\hline Mean & 0.64 & 0.57 & 0.57 & 0.74 & 0.79 & 0.79 \\
\hline SD & 0.39 & 0.32 & 0.32 & 0.32 & 0.32 & 0.32 \\
\hline Median change & -0.13 & -0.05 & -0.18 & 0.10 & 0.04 & 0.14 \\
\hline Change (\%) & $-15.24 \%$ & $-9.35 \%$ & $-21.9 \%$ & $16.40 \%$ & $6.87 \%$ & $22.52 \%$ \\
\hline Wicoxon signed ranks test $\mathrm{p}$ value & 0.003 & 0.003 & 0.001 & 0.001 & 0.002 & $<0.001$ \\
\hline 95\% Confidence Interval & -0.022 to 0.491 & 0.023 to 0.119 & 0.028 to 0.582 & -0.148 to -0.063 & $-0.085,-0.020$ & $-0.213,-0.103$ \\
\hline
\end{tabular}

Percentage changes are shown and changes significant at the $99 \%$ level are shown in bold $(n=18)$ NS=without stimulation; $S=$ with stimulation. 
independently. Intrinsic stiffness of the muscles is likely to be a factor in certain subjects.

\section{Discussion}

This is a small study and these findings should therefore be regarded as preliminary observations that need to be extended. The patients were enthusiastic participants in a clinical trial of FES, other patterns of activation and response might be discovered in a larger sample more representative of hemiplegia after stroke. It should also be recognised that individual patient's use of stimulation varied and was not independently measured. In some cases a poor clinical response may simply have been due to ineffective application of the stimulator, whereas in others, a strong determination to succeed may have enabled them to use the stimulator with a consistency that the average patient might not be able to achieve.

In some cases the rapid stretch of the calf muscles during ankle dorsiflexion may itself have triggered a stretch response. This may have been why using a gradual rise in stimulation amplitude (ramp), after heel switch initiation that allowed the ankle to dorsiflex more slowly, sometimes resulted in a more effective movement. Certain patients showed increased stiffness in the calf muscles to passive stretch, which seemed to reflect intrinsic stiffness of the tissues rather than neuromuscular activation, a phenomenon that has been extensively studied by Dietz and Berger ${ }^{15}$

This study would have been further strengthened if two technical difficulties had been overcome. The first problem was that it was not possible to obtain acceptable recordings of EMG during stimulation because of the stimulus artefact. It is probable that stimulation of the lateral popliteal nerve concurrently activates the tibialis anterior and inhibits the calf muscles. ${ }^{16} 17$ The size of such an effect might constitute a further predictor variable but it could not be demonstrated. The second was the question of whether coactivation in the calf was a stretch response or not. This could have been investigated further by using the strategy of setting up a comparable isometric tracking task as first reported by Hassan, ${ }^{14}$ but it was not feasible to add this to an already very time consuming set of tests. Analysis of the relative roles of the soleus and gastrocnemius muscles would also be of interest, given the evidence that their relative levels of activation in normal subjects tends to shift as the velocity of ankle plantarflexion increases. ${ }^{14}$

\section{APPLICATION TO PHYSIOTHERAPY}

An interesting finding was made during the period in which subjects were learning to perform the tracking task. Initially normal subjects demonstrated some coactivation, which disappeared, as they became skilled at following the signal, a phenomenon that is well described. ${ }^{18}$ In some hemiplegic patients, by contrast, coactivation increased as they practised the task and concurrently their performance tended to decline. This too is commonly reported among physiotherapists who encour- age patients showing this pattern of activity to refrain from making maximal voluntary effort to achieve an optimum level of performance. Whereas it is logical to avoid intensive muscular exercise for those with inappropriate overactivity, such exercise could be beneficial for those whose primary problem is of weakness of muscular activation. Furthermore, those patients in whom stiffness in the absence of an excessive stretch response is identified as a predominant abnormality might benefit more from passive stretching than those in whom the stiffness is primarily due to non-habituating stretch responses. It may be important in the latter case for passive movements to be applied slowly to optimise both comfort and stretch of the soft tissues.

The techniques described in this study could be very useful in exploring the possibilities further and providing a stronger neurophysiological rationale for physiotherapy in this common condition.

We acknowledge the engineering and technical support given by Dr Duncan Wood, Mr Paul Taylor, Dr Steven Crook, Dr Ian Swain, Mr Sean Hagan, and Mr Malcolm Burnett throughout the study. In addition we thank the patients and healthy volunteers who took part.

1 Liberson WT, Holmquest HJ, Scott MED. Functional electrotherapy: stimulation of the common peroneal nerve synchronised with the swing phase of gait of hemiplegic subjects. Arch Phys Med Rehabil 1961;42:101-5.

2 Stefancic M, Rebersek M, Merletti R. The therapeutic effects of the Ljubljana functional electronic brace. Europa Medicophysica. 1976;12:1-9.

3 Bogataj U, Gros N, Malezic M, et al. Restoration of gait during two to three weeks of therapy with multi-channel electrical stimulation. Phys Ther 1989;69:5.

4 Karsnia A. Dillner S, Ebefors I, et al. Why patients use or reject a peroneal muscle stimulator. Advances in External Control of Human Extremities. 1990;251-60.

5 Malezic M, Gros N, Bogataj U. Application of a programmable dual-channel adaptive electrical stimulation system for the control and analysis of gait. F Rehabil Res Dev 1992; 29:41-53.

6 Merletti R, Andina A, Galante M. Clinical experience of electronic peroneal stimulators in 50 hemiparetic patients. Scand F Rehabil Med 1979;11:111-21.

7 Granat MH, Maxwell DJ, Ferguson ACB, et al. Evaluation of common peroneal stimulation for the correction of dropped foot in hemiplegia. Arch Phys Med Rehabil 1996;77:19-24.

8 Glanz M, Klawanskey S, Stason W, et al. Functional electrostimulation in post stroke rehabilitation: a meta analysis of
the randomized controlled trials. Arch Phys Med Rehabil 1996;77:549-53

9 Burridge JH, Taylor PN, Swain ID. The effect of common peroneal stimulation on the effort and speed of walking. A randomised controlled trial with chronic hemiplegic subjects. Clinical Rehabilitation 1997;11:201-10.

10 Knutsson E, Martensson A. Dynamic motor capacity in spastic paresis and its relation to prime mover dysfunction, spastic paresis and its relation to prime mover dysfunction,
spastic reflexes and anatagonist co-activation. Scand $\mathcal{f}$ spastic reflexes and anatago

11 McLellan DL. Co-contraction and stretch reflexes in spasticity during treatment with baclofen. $f$ Neurol Neurosurg Psychiatry 1977;40:30-8

12 McLellan DL, Hassan N, Hodgson JA. Tracking tasks in the assessment of spasticity. In: Delwaide PJ, Young RR, eds.Clinical Neurophysiology in Spasticity. Amsterdam: Elsevier, 1985.

13 Nene AV. Physiological cost index of walking in able-bodied adolescents and adults. Clinical Rehabilitation 1993;7: 319326.

14 Hassan NH. Muscle activation during, repetitive movements in normal and spastic man [doctoral thesis]. Southampton University, 1983

15 Dietz V, Berger W. Normal and impaired muscle stiffness in gait: a new hypothesis about muscle hypertonia. Exp Neurol 1983;79:680-7.

16 Apkarian JA, Naumann S. Stretch reflex inhibition using electrical stimulation in normal subjects and subjects with spasticity. F Biomed Eng 1990;13:67-73.

17 Crone C, Nielsen J, Petersen N, et al. Disynaptic inhibition of ankle extensors in spastic patients. Brain 1994;117: 1161-8.

18 Schmidt RA. Motor control and learning A behavioural emphasis. 3rd ed. Champaign Illinois: Human Kinetics, 1998 ISBN 0-87322-115-X. 